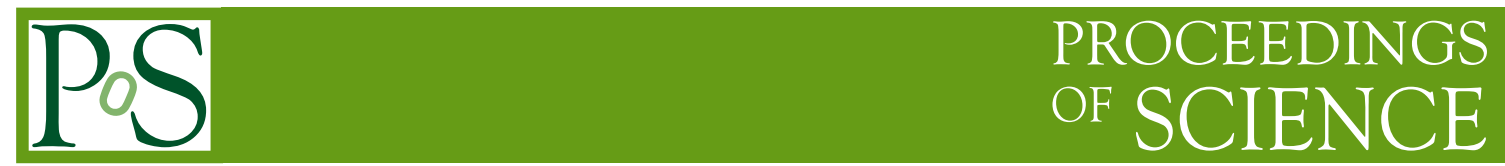

\title{
Results of LHC-ATLAS 8TeV Run
}

\section{Makoto Tomoto*}

Graduate School of Science and Kobayashi-Maskawa Institute, Nagoya University, Japan

E-mail: makoto@hepl.phys.nagoya-u.ac.jp

The results of physics at the LHC-ATLAS experiment, in particular measurements of the properties of a Higgs boson, are presented, based on proton-proton collision data collected in 2011 and 2012. The production strengths and the couplings are measured using decays of the new boson into $\gamma \gamma, Z Z^{*} \rightarrow 4 \ell$, and $W W^{*} \rightarrow \ell v \ell v$. The spin- 0 and CP-even $\left(J^{P}=0^{+}\right)$hypothesis of the standard model (SM) Higgs boson is compared to several alternative hypotheses with $J^{P}=0^{-}$, $1^{+}, 1^{-}, 2^{+}$using decays of the new boson into $\gamma \gamma, Z Z^{*} \rightarrow 4 \ell$, and $W W^{*} \rightarrow e v \mu \nu$. The direct measurement of the Higgs couplings to fermions are performed by using $H \rightarrow b \bar{b}$ and $H \rightarrow \tau \tau$ decay channels. The measured properties are compatible with the SM Higgs boson.

KMI International Symposium 2013 on ăĂIJQuest for the Origin of Particles and the Universe", 11-13 December, 2013

Nagoya University, Japan

${ }^{*}$ Speaker. 


\section{Introduction}

The ATLAS [1] at the LHC recorded $5 \mathrm{fb}^{-1}$ of $7 \mathrm{TeV}$ and $20 \mathrm{fb}^{-1}$ of $8 \mathrm{TeV}$ proton-proton collision data in 2011 and 2012, respectively. The run in this period is called LHC Run I. The performance of the ATLAS detector is understood well. The ATLAS measures production crosssections, masses and other properties of the Standard Model (SM) particles precisely. Finally ATLAS has discovered the Higgs boson in 2012. After the discovery of the Higgs boson, our interests have been focused on looking for the new physics beyond the SM, which can be achievable from the precise measurement of the Higgs boson property and direct search for the new particles.

More than 270 papers including SM measurements, Higgs physics, and new physics searches have been published by the ATLAS collaboration so far. In this article, the contributions of the Nagoya group to the ATLAS experiment is described briefly followed by the measurements of the Higgs boson properties.

\section{Contributions of the Nagoya group}

Nagoya group has joined the ATLAS collaboration since 2006. We make a major contribution to installation, commissioning, and operation of the level one end-cap muon trigger system performed by the thin gap chambers (TGC). Students, postdocs and faculty staffs play a major role in the experts of the trigger system. Our contributions helped, for example, to collect the candidates of the Higgs boson decaying into 4 muons . We also contribute to the physics analysis, especially, the measurements of the top quark pair production cross-section using the di-lepton final state. We would like to extend our research area to the Higgs boson physics and searches for the new physics beyond the SM from the top quark physics.

\section{Measurements of the Higgs boson properties}

One year has passed since a new boson with a mass of $m_{H} \simeq 125.5 \mathrm{GeV}$ was discovered by the ATLAS and the CMS Collaborations [2,3]. Since then our interests have been focused on whether it provides masses to the fermions and bosons and whether it is a Higgs boson of $J^{P}=0^{+}$ as predicted by the standard model (SM). They are addressed experimentally by measuring its properties such as the production strengths, the couplings and the spin-parity. We also search for signs of physics beyond the SM (BSM) by studying its properties.

At the LHC, the Higgs boson is produced via gluon fusion ( $\mathrm{ggF}$ ), vector boson fusion (VBF), associated production with $W$ or $Z(\mathrm{VH})$, and associated production with a top quark pair (ttH). In these mechanisms, $\mathrm{VBF}$ and $\mathrm{VH}$ provide a direct probe of the vector boson coupling while ggF provides an indirect probe of the Higgs-fermion coupling. The Higgs boson with a mass of $m_{H} \simeq 125.5 \mathrm{GeV}$ is detectable at LHC experiments using the $H \rightarrow \gamma \gamma$ via a $W$ boson or a top quark loop, $H \rightarrow Z Z^{*}, H \rightarrow W W^{*}, H \rightarrow \tau \tau$, and $H \rightarrow b \bar{b}$ decay channels.

The expected number of signal events in the studies of the $H \rightarrow \gamma \gamma, H \rightarrow Z Z^{*}$, and $H \rightarrow W W^{*}$ decay channels are around 450,15, and 180 respectively. Using these decay channels, measurements of the properties of the new boson such as the signal strengths, the couplings and the spinparity in the analyses of these three channels based on the $7 \mathrm{TeV}$ and $8 \mathrm{TeV}$ data $[4,5]$ are per- 

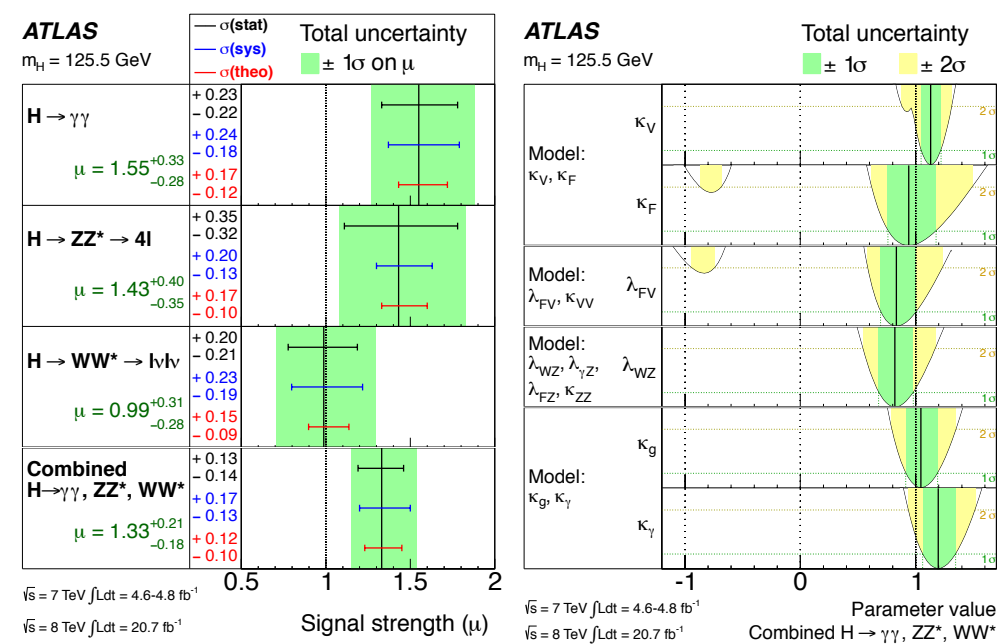

Figure 1: Left: The measured production strengths for a Higgs boson with mass $m_{H}=125.5 \mathrm{GeV}$, normalized to the SM expectations, for diboson final states and their combination [4]. The best-fit values are shown by the solid vertical lines. The total $\pm 1 \sigma$ uncertainty is indicated by the shaded band, with the individual contributions from the statistical uncertainty (top), the total systematic uncertainty (middle), and the theory uncertainty (bottom) on the signal cross-section shown as superimposed error bands. Right: Summary of the measurements of the coupling scale factors (the measurements of $\kappa_{V}$ V.s. $\kappa_{F}$ and $\kappa_{g}$ V.s. $\kappa_{\gamma}$ described in the text together with the ratio of couplings to fermions and vector bosons and the ratio of couplings to $W$ and $Z$ ) for a Higgs boson with mass $m_{H}=125.5 \mathrm{GeV}$ [4]. The best-fit values are represented by the solid vertical lines with the $\pm 1 \sigma$ and $\pm 2 \sigma$ uncertainties given by the dark- and light-shaded bands, respectively.

formed. The direct measurement of the Higgs couplings to the fermions are performed by using $H \rightarrow b \bar{b}$ and $H \rightarrow \tau \tau$ decay channels [6,7] .

\subsection{Production strength and coupling measurements}

The Higgs boson production strengths $\mu$ normalized to the SM expectation (so that $\mu=1$ corresponds to the SM Higgs boson hypothesis and $\mu=0$ to the background-only hypothesis) are measured using the $H \rightarrow \gamma \gamma, H \rightarrow Z Z^{*}$, and $H \rightarrow W W^{*}$ final states. The parameter $\mu$ is determined from a fit to the data using the profile likelihood ratio for a fixed mass hypothesis corresponding to the measured value $m_{H}=125.5 \mathrm{GeV}$. The left-hand figure of Figure 1 shows the production strengths for three channels and their combination. The production strengths are categorized by the vector-boson mediated processes, $\mathrm{VBF}$ and $\mathrm{VH}$, and the gluon mediated processes, ggF and $\mathrm{ttH}$ $\left(\mu_{\mathrm{VBF}+\mathrm{VH}}=\mu_{V B F}=\mu_{\mathrm{VH}}, \mu_{\mathrm{ggF}+\mathrm{ttH}}=\mu_{\mathrm{ggF}}=\mu_{\mathrm{ttH}}\right)$. To test the sensitivity to VBF production alone, the data are fitted with the ratio $\mu_{\mathrm{VBF}} / \mu_{\mathrm{ggF}+\mathrm{ttH}}$. A value $\mu_{\mathrm{VBF}} / \mu_{\mathrm{ggF}+\mathrm{ttH}}=1.4_{-0.3}^{+0.4}(\mathrm{stat})_{-0.4}^{+0.6}(\mathrm{sys})$ is obtained from the combination of the three channels. This result provides evidence at the $3.3 \sigma$ level that a fraction of Higgs boson production occurs through the VBF.

Following the approach and benchmarks recommended in [8], the coupling scaling factors $\kappa_{j}$ are defined in such a way that the cross sections $\sigma_{j}$ and the partial decay width $\Gamma_{j}$ associated with the SM particle $j$ scale with $\kappa_{j}^{2}$ compared to the SM prediction. The results are extracted from fits to the data using the profile likelihood ratio, where the $\kappa_{j}$ couplings are treated either as parameters of interest or as nuisance parameters, depending on the measurement. A number of assumptions 


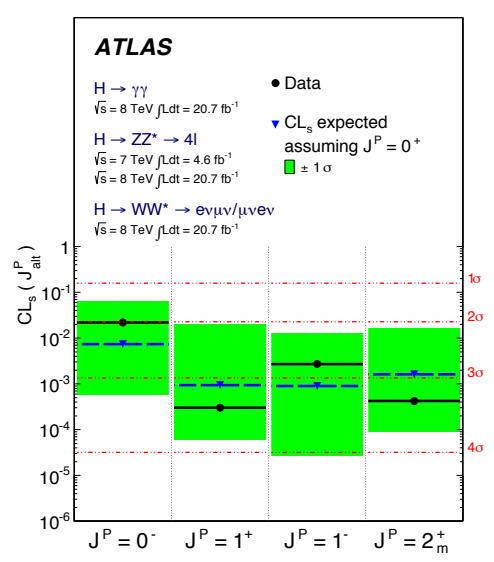

Figure 2: Expected (triangles/dashed lines) and observed (circles/solid lines) $\mathrm{CL}_{\mathrm{S}}$ for alternative spin-parity hypotheses assuming a $J^{P}=0^{+}[5]$. The bands represent the $68 \% \mathrm{CL}_{\mathrm{S}}\left(\mathrm{J}_{\text {alt }}^{\mathrm{P}}\right)$ expected exlusion range for a signal with assumed $J^{P}=0^{+}$.

are made, as described below.

The first benchmark considered assumes one coupling scale factor for fermions $\left(\kappa_{F}\right)$ and one for vector bosons $\left(\kappa_{V}\right)$. The effective scale factors in loop-induced processes (i.e. $\kappa_{\gamma}$ and $\kappa_{g}$ ) and the total Higgs boson width scale factor $\kappa_{H}$ are expressed as a function of the SM coupling scale factors and depend only on $\kappa_{F}$ and $\kappa_{V} . \kappa_{F}$ and $\kappa_{V}$ are measured using the data for the three channels and their combination, assuming $\kappa_{V}>0$ (only the relative sign of $\kappa_{F}$ and $\kappa_{V}$ is physical). Because of the negative interference between the W-boson loop and the top-quark loop in $H \rightarrow \gamma \gamma$ decay, $\kappa_{F}$ prefers the positive relative sign. The $68 \%$ confidence level (CL) intervals of $\kappa_{F}$ and $\kappa_{V}$ are $\kappa_{F} \in[0.76,1.18]$ and $\kappa_{V} \in[1.05,1.22]$, respectively. The second benchmark considered probes the BSM contributions in the $\kappa_{g}$ and $\kappa_{\gamma}$ assuming the couplings of the known particles to the Higgs boson and $\kappa_{H}$ equal to 1 . The best-fit values are $\kappa_{g}=1.04 \pm 0.14$ and $\kappa_{\gamma}=1.20 \pm 0.15$.

The right-hand figure of Figure 1 shows the summary of the measurements of the coupling scale factors for a Higgs boson with $m_{H}=125.5 \mathrm{GeV}$. The compatibility of the best-fit values and SM predictions is between $12 \%$ to $20 \%$.

\subsection{Spin-parity measurements}

Several alternative specific models, $J^{P}=0^{-}, 1^{+}, 1^{-}, 2^{+}$, are tested against the SM Higgs boson $J^{P}=0^{+}$hypothesis, using angular and kinematic distributions in $H \rightarrow \gamma \gamma, H \rightarrow Z Z^{*} \rightarrow 4 \ell$, and $H \rightarrow W W^{*} \rightarrow e v \mu \nu$ decays. The $J=1$ particle decaying to $\gamma \gamma$ is ruled out because of LandauYang theorem $[9,10]$. For $J^{P}=2^{+}$, the graviton-inspired model with minimal coupling to SM particles $\left(2_{m}^{+}\right.$model $)$is chosen as a benchmark [11]. Figure 2 shows expected and observed $\mathrm{CL}_{\mathrm{s}}$ for alternative spin-parity hypotheses assuming a $J^{P}=0^{+}$signal. For the spin-2 hypothesis, the result for the specific $2_{m}^{+}$model is shown. $J^{P}=0^{-}, 1^{+}, 1^{-}$, and $2^{+}$are excluded against $J^{P}=0^{+}$at more than $97.8 \% \mathrm{CL}$. 


\subsection{Direct measurements of Higgs coupling to fermions}

The Higgs coupling to the bottom quark is measured using the VH production, where the $W$ or $Z$ decays leptonically and the Higgs decays to $b \bar{b}$. We search for the $Z H \rightarrow \ell \ell b \bar{b}, W H \rightarrow \ell v b \bar{b}$, and $Z H \rightarrow v v b \bar{b}$, using the final state of the $b \bar{b}$ plus 2 leptons, 1 lepton, or large missing transverse energy, respectively. The best fit of the production strength for the combinations among these channels is measured to be $\mu=0.2_{-0.6}^{+0.7}$.

The Higgs coupling to the $\tau$ lepton is measured using both hadronic and leptonic tau decays. To reduce the QCD backgrounds, events are categorized as "VBF" which is selected by $\tau \tau$ plus dijets with large rapidity gap, and "Boosted" which is selected by $\tau \tau$ with large transverse momentum of the di- $\tau$ system. The multivariate analysis, the boosted decision tree, is used to discriminate the signal from the backgrounds. The production strength for this channel is measured to be $\mu=$ $1.4_{-0.4}^{0.5}$. The observed and expected significance correspond to $4.1 \sigma$ and $3.2 \sigma$, respectively.

\section{Conclusion}

LHC-Run I ends with a great success. The SM physics has been precisely measured and understood. A Higgs-like resonance at $125.5 \mathrm{GeV}$ has beed discovered. All measured properties of this are compatible with the SM Higgs boson, so far. The large area of the new physics parameter space has beed excluded.

LHC will increase the energy and integrated luminosity in the upcoming runs. More precise measurements will be achieved to challenge the SM predictions and will give us hints of the BSM. The energy increase may allow us to discover of the new particles and new phenomena beyond the SM.

\section{References}

[1] ATLAS Collaboration, 2008 JINST 3 S08003.

[2] ATLAS Collaboration, Phys. Lett. B 716 (2012) 1-29.

[3] CMS Collaboration, Phys. Lett. B 716 (2012) 30-61.

[4] ATLAS Collaboration, Phys. Lett. B 726 (2013) 88-119.

[5] ATLAS Collaboration, Phys. Lett. B 726 (2013) 120-144.

[6] ATLAS Collaboration, ATLAS-CONF-2013-079.

[7] ATLAS Collaboration, ATLAS-CONF-2013-108.

[8] LHC Higgs Cross Section Working Group, arXiv:1209.0040 [hep-ex].

[9] L. D. Landau, Dokl. Akad. Nauk Ser. Fiz. 60 (1948) 207.

[10] C.-N. Yang, Phys. Rev. 77 (1950) 242.

[11] Y. Gao, A.V. Gritsan, Z. Guo, K. Melnikov, M. Schulze, and N.V. Tran, Phys. Rev. D 81, 075022 (2010). 